\title{
STUDY OF HOW CLINICAL AND SOCIODEMOGRAPHIC VARIABLES INFLUENCE CHEMOTHERAPY-INDUCED COGNITIVE DETERIORATION IN WOMEN WITH BREAST CANCER
}

\author{
Blanca Rodríguez Martín 1, Eduardo José Fernández Rodríguez 1,2,5,*, Celia Sanchez Gomez 3,5, Juan Jesús Cruz Her- \\ nández 14,5 and María Isabel Rihuete Galve 1,2,5
}

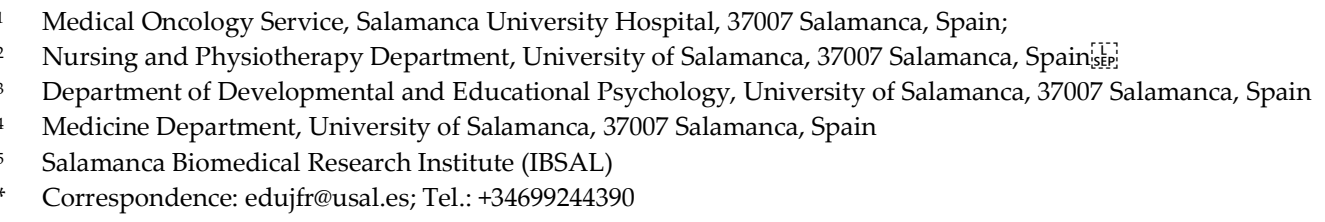

Abstract: Background: Of the many side effects suffered by cancer patients, those related to cognitive performance have become increasingly prominent in clinical practice. We know that chemotherapy generates a series of side effects, such as nausea and vomiting, alopecia, and so on, which can be counteracted by complementary medication. However, in the case of post-chemotherapy cognitive impairment, or chemo brain, these cannot be controlled with drug therapies. However, before any intervention can be considered, it is necessary to know exactly what cognitive impairment is being triggered. For this reason, we decided to study the cognitive status of breast cancer patients. Methods: analytical, prospective, three-measure longitudinal, intrasubject unifactorial, nonprobabilistic and accidental assignment study. The sample came from the Medical Oncology Department at Hospital de Salamanca, in Spain. Cognitive function (Trail Making Test and Stroop Test) was established as the primary variable; the presence of sleep disorders (Insomnia Severity Index, ISI) and anaemia (haemoglobin levels in blood) were analysed as secondary variables, in addition to sociodemographic variables (age, stage, type of carcinoma, radiotherapy, menopause, social support network, marital status, years of schooling and employment status). Results: We recruited 151 individuals according to the selection criteria. We can confirm that factors including anaemia, menopause, patient support network and marital status, years of schooling, and employment status did affect the cognitive performance of the patients in active chemotherapy treatment in the study. In contrast, sleep disorders, age, radiotherapy treatment, stage of disease, and type of carcinoma did not affect the cognitive performance of the cancer patients. Conclusions: Chemotherapy does impact the cognitive performance of breast cancer patients.

Keywords: Chemobrain; Cancer; Cognitive function; Insomnia.

\section{Introduction}

Of the many symptoms suffered by cancer patients, cognitive performance has become increasingly prominent in clinical practice, due to increased survival rates and concern about patient quality of life.

The studies reviewed indicate that chemotherapy-induced cognitive impairment may occur in $15-50 \%$ of cancer patients [1-4], whereas stereotactic radiosurgery (LITT) seems not to affect neurocognitive function [5-6].

The majority of studies on cognitive functioning have been conducted in women with breast cancer, as this is a large population, and the patients tend to be functionally healthier and have fewer symptoms than patients with other oncological pathologies [4]. [7-10].

In addition, the high survival rates of these patients explain how important cognitive problems may be for them

We know that chemotherapy generates a series of side effects that include nausea and vomiting, alopecia, anaemia, immunosuppression, altered taste, diarrhoea, constipation, mucositis, decreased sexual desire, loss of sensation, and so forth [1-4]. These effects are currently counteracted by complementary medication with the aim of maintaining the patients' quality of life as far as possible. However, cognitive side effects, more commonly known as chemo brain, cannot be controlled through pharmacological therapies.

Indeed, before focusing on possible interventions, it is necessary to determine the exact cognitive impairment caused by the effects of chemotherapy treatments. 
Most studies state the need for pre-treatment assessment of cognitive functioning in order to establish a baseline against which later results can be compared, once chemotherapy treatment is finished [9-14].

This first assessment allows the true magnitude of the change observed after cancer treatment to be studied accurately, in turn allowing its evolution over time to be established. To ascertain the levels of cognitive deterioration in oncology patients prior to treatment, and any subsequent post-therapy increase, which is maintained even once the treatment has ended, we decided to perform a longitudinal study that would allow us to verify the veracity of these assumptions.

We also considered it necessary to analyse the influence of other symptoms and characteristics of the study subjects, which could have a bearing on this cognitive deterioration, such as sleep-wake disturbances, anaemia, the menopause, and certain social factors including family responsibilities in terms of caring for children or elderly people in their care.

On the basis of the above considerations, we formulated the following hypothesis: throughout the course of their treatment, patients with locoregional breast cancer will experience cognitive deterioration that will persist for at least two months after the end of treatment.

\subsection{OBJECTIVES:}

The main objective was to study the cognitive status of breast cancer patients at the Complejo Asistencial Universitario de Salamanca (CAUSA) group of hospitals.

Our specific objectives were:

- To determine whether sleep disorders and anaemia affect the cognitive performance of patients.

- To study whether cognitive performance is age-related.

- To study whether radiation therapy as an adjuvant treatment to chemotherapy and the stage of the disease increases the risk of cognitive impairment.

- To analyse whether the type of breast carcinoma influences the cognitive performance of patients.

- To assess whether menopause influences the cognitive performance of patients.

- To study whether sociodemographic variables -support network, years of schooling, employment status at the time of measurement and marital status- influence the cognitive performance of patients.

\section{Materials and Methods}

Study design: an analytical, prospective, three-measure longitudinal, intrasubject unifactorial, non-probabilistic and accidental assignment study was conducted.

Participants: the sample was taken from the CAUSA Medical Oncology Service.

Selection criteria: a series of inclusion, exclusion, and withdrawal criteria were established, as shown in Figure 1 . 


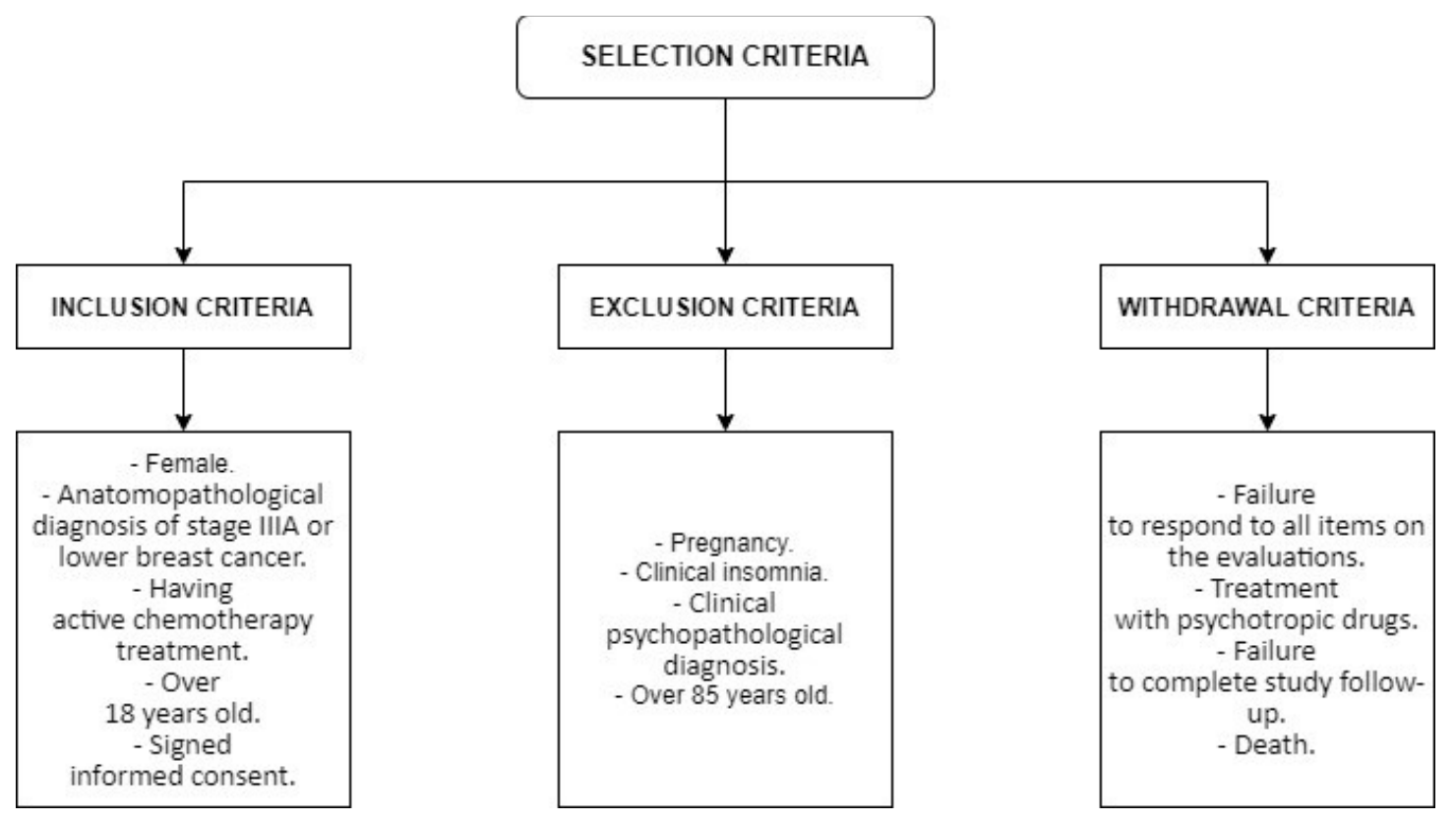

Figure 1. Selection criteria.

Sample size: a convenience sample was established. Patients were randomly assigned to the study between October 2015 and January 2017.

Description of variables.

Cognitive function (processing speed and attention suddenly) was established as the primary variable, while the presence of sleep disorders and anaemia were analysed as secondary variables. A series of intervening variables were also established: age, disease stage, type of carcinoma, radiotherapy, menopause, social support network, marital status, years of schooling, and employment status.

Assessment tools:

- The Trail Making Test (TMT) and Stroop test: to assess cognitive function. These tests were chosen over others as we believe they are the most appropriate to assess cognitive status in people without clinically diagnosed cognitive impairment.

- The Insomnia Severity Index (ISI): to assess the presence of sleep disorders.

- Blood haemoglobin levels: to assess the presence of anaemia.

The other variables were collected from the clinical history and a questionnaire created exclusively for the study.

The data was collected between October 2015 and January 2017.

Procedure:

To carry out this research, an investigative research project was drafted and submitted to the CAUSA ethics committee for a feasibility review. Once it had been approved, patient recruitment began. A baseline was established at the start of chemotherapy treatment. Those patients who verbally agreed to participate in the study were invited to the medical oncology clinic to sign the informed consent form and to receive an explanation of the study objectives and procedures. The patients were then recruited to the study according to the aforementioned selection criteria.

All the assessment procedures were carried out on the same day that a patient was undergoing their chemotherapy treatment, in the time between the analysis and the start of the cycle.

In the first interview, the patients' clinical and sociodemographic data were collected and baseline information was gathered using the selected tests.

The progress of the patients was monitored after three months of chemotherapy, and again two months after the treatment had been completed. 
Three measurement points were therefore established through which to study the progress of cognitive performance: at the start of chemotherapy, at the initial evaluation; three months after treatment had been initiated, at the follow-up evaluation; and, finally, two months after the end of treatment, at the final evaluation.

These three measurement points were established to assess the effect chemotherapy had on the cognitive performance of the patients being studied. In total, 613 interventions were performed, lasting approximately one hour each.

Ethical issues:

The study was conducted with the authorisation of the Clinical Research Ethics Committee of the Salamanca Health Area (ID:0000263), with the prior informed consent of the study subjects and in accordance with the Declaration of Helsinki. The participants were informed of the objectives of the project and the risks and benefits of the interventions that were carried out. Subject confidentiality was guaranteed at all times in accordance with the laws on the protection of personal data and biomedical research as established in the provisions of Organic Law 3/2018, of 5 December, on the Protection of Personal Data and guarantee of digital rights and Regulation (EU) 2016/679 of the European Parliament and of the Council of 27 April, 2016, on data protection (GDPR), and under the conditions established by Law 14/2007 on biomedical research. Name of the Council: Comité de Bioética de la Universidad de Salamanca (Bioethics Committee of the University of Salamanca). Board Affiliation: University of Salamanca.

Statistical analysis.

The statistical analysis was pre-planned, with minor modifications being implemented once the study had been conducted. Initially, the information was thoroughly reviewed and filtered to detect possible data-collection errors, and so that the exclusion criteria established for the study could be correctly and accurately applied. To this end, we used a descriptive analysis, focusing on the maximum and minimum values obtained for the quantitative variables, as well as on the presence of possible outliers in the box plots.

Descriptive statistics: the study variables were analysed using the Shapiro-Wilk and Kolmogorov-Smirnov tests to determine the normality of the sample, thereby identifying the way in which to proceed. Once we had established that the variables followed a normal distribution, they were defined according to their means, standard deviations and ranges. Discrete variables were defined by cases and percentages.

Analytical statistics: a number of tests were performed, including Analysis of Variance (ANOVA) Intra or Repeated Measures Test, to determine whether there were significant differences in the variables measuring cognitive impairment between the three points at which the patients were measured (before, during and after chemotherapy).

The statistical software package SPSS 23.0 was used to process the data.

\section{Results}

The distribution of the sample can be seen in Figure 2. Of the 174 patients interviewed, 23 were excluded, 20 of whom were eliminated from the study as they met the withdrawal criteria. The final sample therefore comprised a total of 151 individuals. 


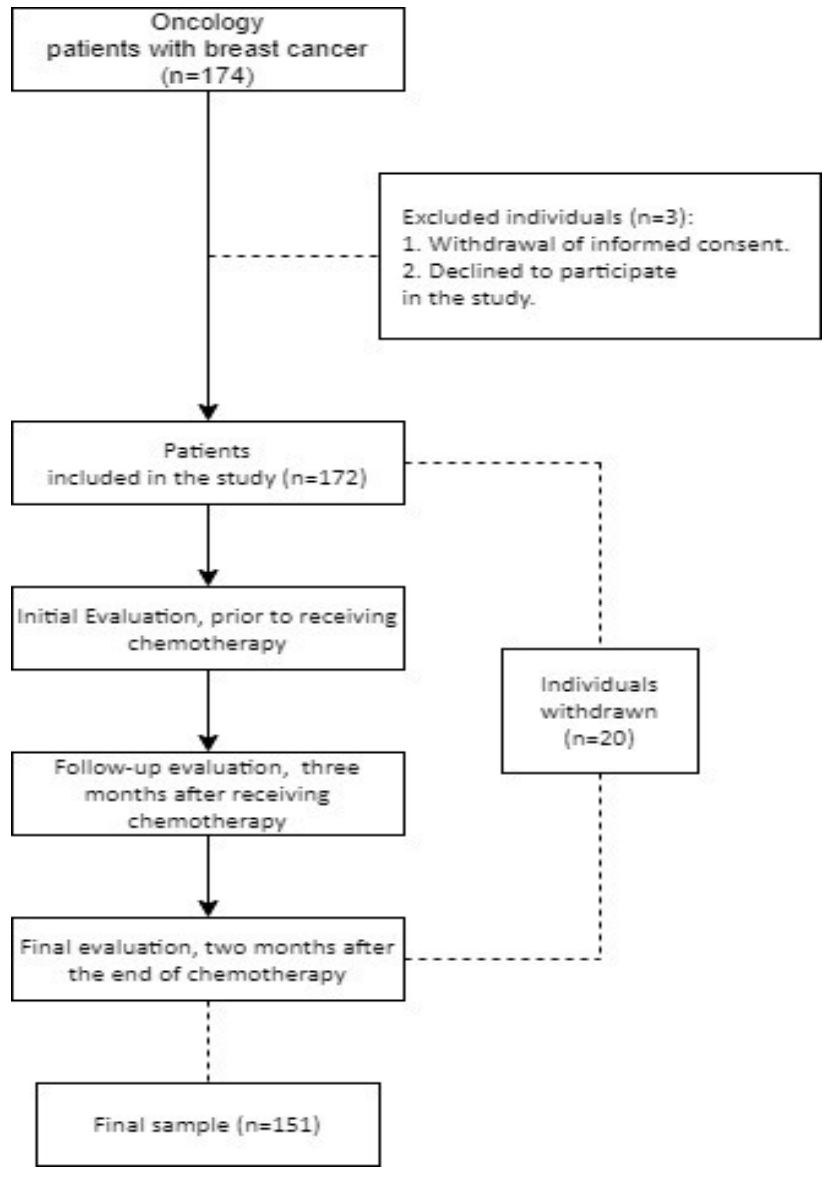

Figure 2. Study Flow Chart.

The first objective was to analyse the impact of chemotherapy on the cognitive domains at the three measurement points studied.

A general pattern emerged for most of the cognitive domains studied: as chemotherapy treatment progressed, the cognitive performance of the patients in the study worsened significantly with respect to their baseline scores.

When observing the evolution of processing speed (measured through a symbol search and number key) and attention (measured using the Trail Making Test (TMT) and Stroop Test(ST)), a decrease was seen throughout the chemotherapy treatment (Symbol search mean=23.41; number key M=38.32; TMT_A M=53.95; TMT_B M=121.42; ST words and colour $\mathrm{M}=43.75)$, with the lowest cognitive performance score two months after the end of the treatment.

The second objective was to determine whether the sleep disorders and anaemia in patients affects their cognitive performance.

\subsection{Sleep Quality.}

After performing a repeated measures ANOVA for independent samples, no significant differences were found between patients who reported poor, fair or good sleep quality either before, during or after chemotherapy treatment in any of the cognitive domains we measured $(\mathrm{p}<0.05)$.

\subsection{Anaemia.}

- Before treatment.

To check whether there were significant differences in cognitive performance in terms of patient haemoglobin level, a repeated measures ANOVA for independent samples was performed.

To facilitate a better understanding of the results, only the variables for which statistically significant differences were found between the groups will be shown. 
The differences between the women classified in the groups were significant: (1) low haemoglobin level (less than $11 \mathrm{~g} / \mathrm{dL})$; (2) medium (11-16 g/dL); and (3) high (more than $16 \mathrm{~g} / \mathrm{dL}$ ) in the following tests used to assess cognitive performance:

- TMT_A before $(\mathrm{F}=3.642 ; \mathrm{p}<0.05)$,

- TMT_A during $(\mathrm{F}=3.632 ; \mathrm{p}<0.05)$,

- TMT_A after $(\mathrm{F}=3.508 ; \mathrm{p}<0.05)$,

- TMT_B before $(\mathrm{F}=5.945 ; \mathrm{p}<0.01)$,

- $\quad$ TMT_B during $(\mathrm{F}=5.600 ; \mathrm{p}<0.01)$,

- TMT_B after $(F=6.177 ; p<0.01)$.

Scheffé's Post Hoc or Multiple Comparisons test was performed to find out between which groups there were differences in the various cognitive domains (in this case, cognitive deterioration is indicated by high scores). Differences were found between women classified as having low and medium haemoglobin levels (1-2*). In all cases, the group with low haemoglobin levels had cognitive deterioration, i.e., higher scores.

The averages are presented in Table 2.

Table 1. Evolution of the domains studied in the three measurement moments.

\begin{tabular}{|c|c|c|c|c|}
\hline VARIABLES & $\mathbf{M}$ & S.D. & $\begin{array}{c}\text { Minimum score } \\
\text { obtained }\end{array}$ & $\begin{array}{c}\text { Maximum score } \\
\text { obtained }\end{array}$ \\
\hline TMT_A Initial & 50.85 & 16.486 & 27 & 89 \\
\hline TMT_A Follow up & 53.95 & 16.845 & 29 & 91 \\
\hline TMT_A Final & 55.81 & 17.199 & 31 & 95 \\
\hline TMT_B Initial & 116.75 & 32.853 & 65 & 198 \\
\hline TMT_B Follow up & 121.42 & 33.086 & 66 & 69 \\
\hline TMT_B Final & 125.23 & 33.25 & 69 & 209 \\
\hline Stroop palabra Initial & 122.27 & 4.286 & 110 & 132 \\
\hline Stroop palabra Follow up & 117.77 & 3.921 & 108 & 127 \\
\hline Stroop palabra Final & 114.93 & 3.871 & 107 & 126 \\
\hline Stroop color Initial & 76.86 & 4.032 & 70 & 86 \\
\hline Stroop color Follow up & 70.62 & 4.415 & 60 & 81 \\
\hline Stroop color Final & 67.56 & 3.834 & 59 & 79 \\
\hline $\begin{array}{c}\text { Stroop palabra y color } \\
\text { Initial }\end{array}$ & 49.30 & 4.064 & 40 & 59 \\
\hline $\begin{array}{c}\text { Stroop palabra y color } \\
\text { Follow up }\end{array}$ & 43.75 & 3.912 & 33 & 53 \\
\hline Stroop palabra y color Final & 41.94 & 3.077 & 31 & 49 \\
\hline
\end{tabular}

$\mathrm{M}=$ Mean; S.D.=Standard deviation. 
Table 2. Influence of the hemoglobin level in the study tests that were significant before chemotherapy treatment.

\begin{tabular}{|c|c|c|c|c|c|}
\hline \multirow[b]{3}{*}{ VARIABLES } & \multicolumn{5}{|c|}{ Hemoglobin level } \\
\hline & $\begin{array}{l}\text { 1. Low (less } 11 \\
\begin{array}{l}\mathrm{g} / \mathrm{dl}) \\
\mathrm{n}=89\end{array}\end{array}$ & $\begin{array}{l}\text { 2. Medium (11- } 16 \\
\qquad \begin{array}{l}\mathrm{g} / \mathrm{dl}) \\
\mathrm{n}=60\end{array}\end{array}$ & $\begin{array}{l}\text { 3. High (plus } 16 \\
\qquad \begin{array}{c}\mathrm{g} / \mathrm{dl} \text { ) } \\
\mathrm{n}=2\end{array}\end{array}$ & & \\
\hline & $\mathrm{M}$ & $\mathrm{M}$ & $\mathrm{M}$ & Scheffé & $\mathrm{F}$ \\
\hline TMT_A Initial & 53.81 & 46.72 & 43.50 & $1-2^{*} 3$ & $3.642 p<.05$ \\
\hline $\begin{array}{c}\text { TMT_A Follow } \\
\text { up }\end{array}$ & 56.98 & 49.68 & 47.50 & $1-2^{*} 3$ & $3.632 p<.05$ \\
\hline TMT_A Final & 58.82 & 51.63 & 47.00 & $1-2^{*} 3$ & $3.508 p<.05$ \\
\hline TMT_B Initial & 124.17 & 106.33 & 99.00 & $1-2^{*}$ & $5.945 p<.01$ \\
\hline TMT_B Follow up & 128.69 & 111.22 & 104.00 & $1-2^{*}$ & $5.600 p<.01$ \\
\hline TMT_B Final & 132.85 & 114.48 & 108.50 & $1-2^{*}$ & $6.177 p<.01$ \\
\hline
\end{tabular}

M=Mean of the variables. ${ }^{*}$ Significant differences between the two groups in Scheffé's Multiple Comparisons test

- During treatment.

A repeated measures ANOVA for independent samples was performed during the treatment to determine whether haemoglobin levels had affected cognitive performance at this point.

Again, only those variables in which statistically significant differences were found between the groups will be listed.

Significant differences were found in the following domains:

- TMT_A before $(\mathrm{F}=4.532 ; \mathrm{p}<0.01)$,

- TMT_A during $(\mathrm{F}=4.913 ; \mathrm{p}<0.05)$,

- TMT_A after $(\mathrm{F}=4.665 ; \mathrm{p}<0.01)$,

- $\quad$ TMT_B before $(\mathrm{F}=6.167 ; \mathrm{p}<0.01)$,

- TMT_B during $(\mathrm{F}=5.764 ; \mathrm{p}<0.01)$,

- TMT_B after $(\mathrm{F}=6.071 ; \mathrm{p}<0.01)$.

Scheffé's Post Hoc or Multiple Comparisons test was performed to find out between which groups there were differences in the various cognitive domains. Differences were observed between patients classified as having low and medium haemoglobin levels $\left(1-2^{*}\right)$, and the patients with highest cognitive impairment scores were in the group with low haemoglobin levels. The averages are presented in Table 3.

Table 3. Influence of the hemoglobin level in the study tests that were significant during chemotherapy treatment..

\begin{tabular}{|c|c|c|c|c|c|}
\hline \multirow[b]{3}{*}{ VARIABLES } & \multicolumn{5}{|c|}{ Hemoglobin level } \\
\hline & $\begin{array}{l}\text { 1. Low (less } 11 \\
\begin{array}{l}\mathrm{g} / \mathrm{dl} \text { ) } \\
\mathrm{n}=87\end{array}\end{array}$ & $\begin{array}{l}\text { 2. Medium (11- } 16 \\
\mathrm{~g} / \mathrm{dl}) \mathrm{n}=60\end{array}$ & $\begin{array}{l}\text { 3. High (plus } 16 \\
\qquad \begin{array}{l}\text { g/dl) } \\
\mathrm{n}=4\end{array}\end{array}$ & & \\
\hline & $\mathrm{M}$ & $\mathrm{M}$ & $\mathrm{M}$ & Scheffé & é $F$ \\
\hline TMT_A Initial & 53.63 & 46.18 & 60.50 & $1-2^{*}$ & $4.532 p<.01$ \\
\hline $\begin{array}{c}\text { TMT_A Follow } \\
\text { up }\end{array}$ & 56.90 & 49.00 & 64.25 & $1-2^{*}$ & $4.913 p<.05$ \\
\hline TMT_A Final & 58.72 & 50.88 & 66.25 & $1-2^{*}$ & $4.665 p<.01$ \\
\hline TMT_B Initial & 123.68 & 105.68 & 132.00 & $1-2^{*}$ & $6.167 \mathrm{p}<.01$ \\
\hline $\begin{array}{c}\text { TMT_B Follow } \\
\text { up }\end{array}$ & 128.22 & 110.60 & 135.75 & $1-2^{*}$ & $5.764 p<.01$ \\
\hline TMT_B Final & 132.26 & 114.08 & 139.50 & $1-2^{*}$ & $6.071 \mathrm{p}<.01$ \\
\hline
\end{tabular}

$\mathrm{M}=$ Mean of the variables. * Significant differences between the two groups in Scheffé's Multiple Comparisons test. 
- After treatment.

A repeated measures ANOVA for independent samples was performed to determine whether haemoglobin levels affected cognitive performance.

Only the results in which statistically significant differences were found between the groups are shown.

- Stroop word before $(\mathrm{F}=17.884 ; \mathrm{p}<0.001)$;

- Stroop word during $(\mathrm{F}=8.728 ; \mathrm{p}<0.001)$;

- Stroop word after $(\mathrm{F}=5.337 ; \mathrm{p}<0.001)$;

- Stroop colour before $(\mathrm{F}=13.098 ; \mathrm{p}<0.001)$;

- Stroop colour during $(\mathrm{F}=5.179 ; \mathrm{p}<0.01)$;

- Stroop colour after ( $\mathrm{F}=3.498 ; \mathrm{p}<0.05)$;

- Stroop word and colour before (F=11.348; $\mathrm{p}<0.001)$;

- Stroop word and colour during ( $F=9.117 ; \mathrm{p}<0.001)$;

- Stroop word and colour after ( $\mathrm{F}=5.645 ; \mathrm{p}<0.01)$.

As the age variable presented three comparison groups, Scheffé's Multiple Comparisons or Post Hoc test was carried out to find out between which age groups there were differences in the various tests measuring the cognitive domains studied.

It was found that there were significant differences between the three age groups: young people, adults, and older adults. In all cases, the group of older adults (aged 66-80 years) showed greater cognitive deterioration, with higher scores. The averages are presented in Table 4.

Table 4. Influence of age on cognitive performance.

\begin{tabular}{|c|c|c|c|c|c|c|}
\hline \multirow[b]{3}{*}{ VARIABLES } & \multicolumn{6}{|c|}{ Age } \\
\hline & $\begin{array}{c}\text { 1. Young } \\
(n=17)\end{array}$ & $\begin{array}{l}\text { 2. Adult } \\
(n=100)\end{array}$ & $\begin{array}{l}\text { 3. Higher } \\
(\mathrm{n}=34)\end{array}$ & \multirow[b]{2}{*}{ Scheffé } & \multirow[b]{2}{*}{$\mathrm{F}$} & \multirow[b]{2}{*}{$\mathrm{p}$} \\
\hline & $\mathbf{M}$ & $\mathrm{M}$ & $\mathrm{M}$ & & & \\
\hline TMT_A Initial & 32.94 & 44.21 & 79.35 & $1 * 2 * 3 *$ & 844.815 & $\mathrm{p}<.001$ \\
\hline TMT_A Follow up & 35.53 & 47.20 & 83.03 & $1^{*} 2^{*} 3^{*}$ & 828.723 & $\mathrm{p}<.001$ \\
\hline TMT_AA Final & 36.59 & 49.02 & 85.38 & $1^{*} 2^{*} 3^{*}$ & 802.935 & $\mathrm{p}<.001$ \\
\hline TMT_B Initial & 74.71 & 105.57 & 170.65 & $1^{*} 2^{*} 3^{*}$ & 508.042 & $\mathrm{p}<.001$ \\
\hline TMT_B Follow up & 78.47 & 110.29 & 175.62 & $1^{*} 2^{*} 3^{*}$ & 516.188 & $\mathrm{p}<.001$ \\
\hline TMT_B Final & 80.71 & 114.28 & 179.71 & $1^{*} 2^{*} 3^{*}$ & 563.552 & $\mathrm{p}<.001$ \\
\hline Stroop palabra Initial & 122.18 & 123.45 & 118.85 & $1-3^{*}$ y $2-3^{*}$ & 17.884 & $\mathrm{p}<.001$ \\
\hline Stroop palabra Follow up & 116.71 & 118.66 & 115.71 & $2-3^{*}$ & 8.728 & $\mathrm{p}<.001$ \\
\hline Stroop palabra Final & 114.71 & 115.58 & 113.15 & $2-3^{*}$ & 5.337 & $\mathrm{p}<.001$ \\
\hline Stroop color Initial & 77.65 & 77.71 & 73.97 & $1-3^{*}$ & 13.098 & $\mathrm{p}<.001$ \\
\hline Stroop color Follow up & 71.12 & 71.24 & 68.53 & $2-3^{*}$ & 5.179 & $\mathrm{p}<.01$ \\
\hline Stroop color Final & 68.12 & 67.98 & 66.06 & $2-3^{*}$ & 3.498 & $\mathrm{p}<.05$ \\
\hline Stroop palabra y color Initial & 51.76 & 49.72 & 46.82 & $1-3^{*}$ y $2-3^{*}$ & 11.348 & $\mathrm{p}<.001$ \\
\hline Stroop palabra y color Follow up & 45.18 & 44.31 & 41.41 & $1-3^{*}$ y $2-3^{*}$ & 9.117 & $\mathrm{p}<.001$ \\
\hline Stroop palabra y color Final & 41.82 & 42.46 & 40.47 & $2-3^{*}$ & 5.645 & $\mathrm{p}<.01$ \\
\hline
\end{tabular}

$\mathrm{M}=$ Mean of the variables. ${ }^{*}$ Significant differences between the two groups in Scheffé's Multiple Comparisons test.

The next objective was to study whether radiotherapy as an adjuvant treatment to chemotherapy increased the risk of cognitive deterioration.

To study whether radiotherapy increased or worsened the cognitive performance of the patients in the sample, we used the Student's t-test for independent samples. 
No significant differences were found between patients who received radiotherapy and those who did not, in any of the cognitive domains assessed by the tests. Graphically, there were also no significant differences between the scores obtained by women who underwent radiotherapy and those who did not.

The results to assess whether the oncological disease stage influenced the cognitive performance of the patients were obtained after a repeated measures ANOVA for independent samples was conducted.

As the disease stage variable presented three comparison groups, Scheffé's Multiple Comparisons or Post Hoc was used to find out between which groups there were differences in the various cognitive domains studied.

We observed that, in the Digits subtest after treatment, there were differences between Stages I and III*, with greater cognitive deterioration in Stage I patients.

This was in contrast to the results found in TMT_A after $(\mathrm{F}=3.185 ; \mathrm{p}<0.05)$, TMT_B before $(\mathrm{F}=3.958 ; \mathrm{p}<0.05)$, TMT_B during $(\mathrm{F}=3.892 ; \mathrm{p}<0.05)$, TMT_B after $(\mathrm{F}=4.653 ; \mathrm{p}<0.05)$. In these, for the Scheffé test, significant differences were also found between Stages I and III, but, in all cases, the worsened cognitive performance occurred in Stage III.

These higher values for a greater number of tests in Stage III show that patients at this stage exhibited worse cognitive performance than in the other two stages studied.

The next objective we studied was to analyse whether the type of breast carcinoma influenced the cognitive performance of the patients; to do this, we ran a repeated measures ANOVA for independent samples.

The next objective was to assess whether menopause influenced the cognitive performance of patients; we used the Student's t test for independent samples to assess this.

The differences between premenopausal and postmenopausal women in the tests used to assess the cognitive domains were significant.

In all the tests, postmenopausal women had higher scores, in other words, greater cognitive deterioration than premenopausal women. The averages are presented in Table 5.

Table 5. Influence of menopause on cognitive performance.

\begin{tabular}{|c|c|c|c|c|c|c|}
\hline \multirow[b]{3}{*}{ VARIABLES } & \multicolumn{6}{|c|}{ Menopausia } \\
\hline & \multicolumn{2}{|c|}{ PRE (n=83) } & \multicolumn{2}{|c|}{$\operatorname{POST}(\mathrm{n}=68)$} & \multirow[b]{2}{*}{$\mathrm{t}$} & \multirow[b]{2}{*}{$\mathrm{p}$} \\
\hline & $\mathbf{M}$ & S.D & $\mathrm{M}$ & S.D & & \\
\hline TMT_A Initial & 41.64 & 5.628 & 62.10 & 18.332 & -8.869 & $\mathrm{p}<.001$ \\
\hline TMT_A Follow up & 44.28 & 5.579 & 65.76 & 18.429 & -9.273 & $\mathrm{p}<.001$ \\
\hline TMT_A Final & 45.89 & 5.961 & 67.91 & 18.661 & -9.348 & $\mathrm{p}<.001$ \\
\hline TMT_B Initial & 98.88 & 14.527 & 138.56 & 35.774 & -8.585 & $\mathrm{p}<.001$ \\
\hline TMT_B Follow up & 103.11 & 14.795 & 143.76 & 35.508 & -8.834 & $\mathrm{p}<.001$ \\
\hline TMT_B Final & 106.60 & 15.122 & 147.97 & 35.223 & -9.027 & $\mathrm{p}<.001$ \\
\hline Stroop palabra Initial & 123.53 & 4.053 & 120.74 & 4.084 & 4.202 & $\mathrm{p}<.001$ \\
\hline Stroop palabra Follow up & 118.25 & 3.996 & 117.19 & 3.774 & 1.665 & n.s \\
\hline Stroop palabra Final & 115.54 & 4.037 & 114.19 & 3.546 & 2.160 & $\mathrm{p}<.05$ \\
\hline Stroop color Initial & 77.72 & 3.801 & 75.81 & 4.082 & 2.978 & $\mathrm{p}<.01$ \\
\hline Stroop color Follow up & 71.16 & 4.766 & 69.96 & 3.877 & 1.673 & n.s. \\
\hline Stroop color Final & 68.04 & 4.206 & 66.99 & 3.262 & 1.728 & n.s. \\
\hline Stroop palabra y color Initial & 50.81 & 3.918 & 47.46 & 3.453 & 5.513 & $\mathrm{p}<.001$ \\
\hline Stroop palabra y color Follow up & 45.16 & 3.931 & 42.04 & 3.155 & 5.282 & $\mathrm{p}<.001$ \\
\hline Stroop palabra y color Final & 42.70 & 3.027 & 41.01 & 2.899 & 3.466 & $\mathrm{p}<.01$ \\
\hline
\end{tabular}

$\mathrm{M}=$ Mean; S.D=Standard devition. Significant differences between the groups $\mathrm{p} \leq .05 ; \mathrm{p} \leq .01 ; \mathrm{p} \leq .001 ;$ n.s $=$ there are no significant differences between the groups. 
Table 6. Influence of employment status on cognitive performance after completing chemotherapy treatment.

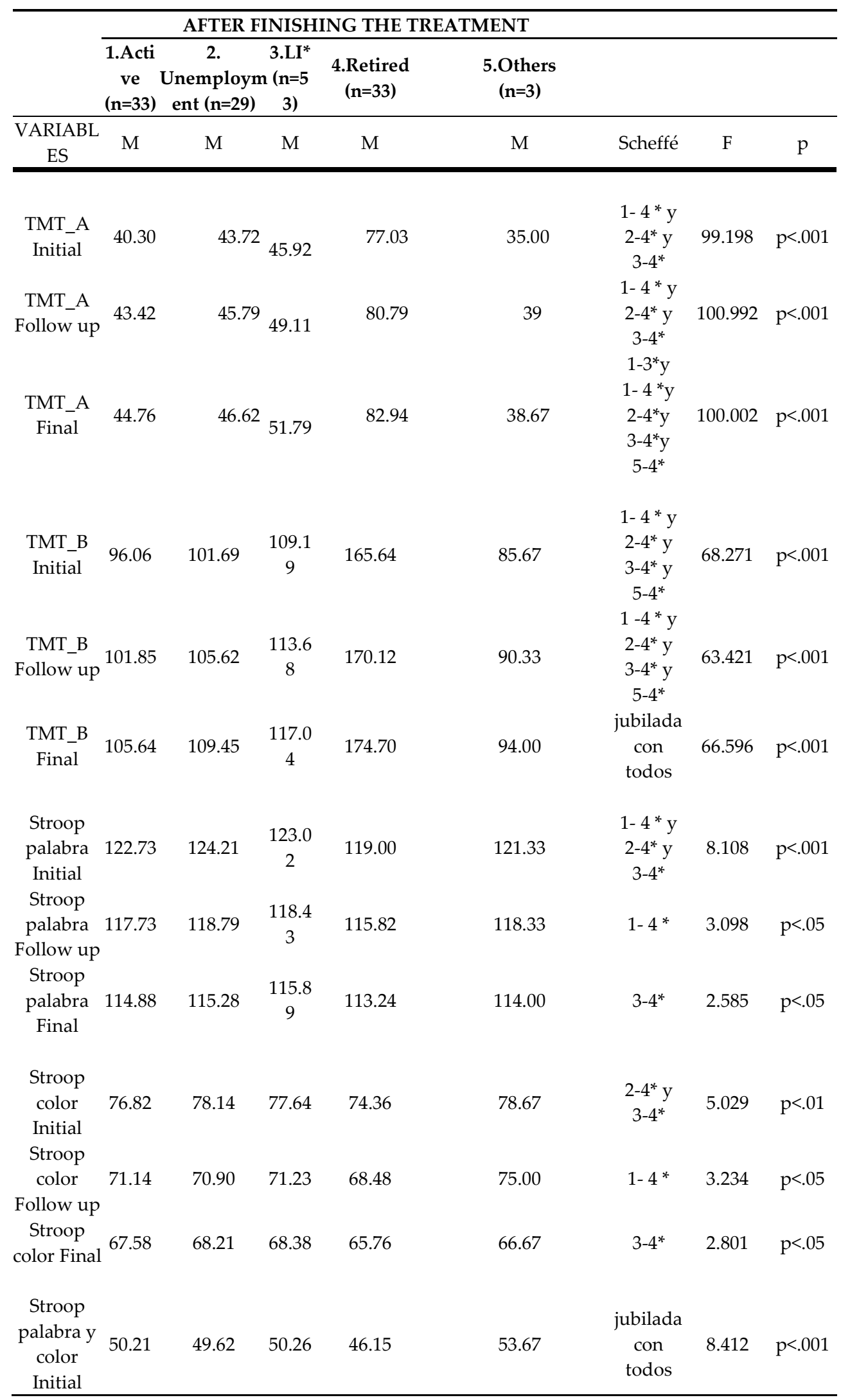




\begin{tabular}{|c|c|c|c|c|c|c|c|}
\hline $\begin{array}{l}\text { Stroop } \\
\text { palabra y } 44.12 \\
\text { color } \\
\text { Follow up }\end{array}$ & 44.59 & 44.89 & 40.85 & 43.67 & $\begin{array}{c}1-4^{*} y \\
2-4^{*} y \\
3-4^{*}\end{array}$ & 7.038 & $\mathrm{p}<.001$ \\
\hline $\begin{array}{l}\text { Stroop } \\
\text { palabra y } 41.76 \\
\text { color Final }\end{array}$ & 42.48 & 42.89 & 40.12 & 41.94 & $3-4^{*}$ & 4.841 & $\mathrm{p}<.01$ \\
\hline
\end{tabular}

The penultimate objective was to study whether sociodemographic variables, support network and marital status, influence the cognitive performance of the patients.

Support network.

After performing the Student's t-test for Independent samples, significant differences were found between those with a good family support network and those with poor support, in the following cognitive performance tests: Stroop Words and Colour after $(t=2.5 ; \mathrm{p}<0.05)$.

Although significant differences were only found in these measures, where it is observed that in Vocabulary after patients with a poor support network $(\mathrm{M}=23.42)$ show more cognitive deterioration than those with a good support network $(\mathrm{M}=27.87)$, and in Stroop Word and Colour after, the patients who have a bad support network $(\mathrm{M}=39.83)$ also present worse cognitive performance than those who have a good support network (M=42.12), there is a trend in most of the tests towards patients with a poor support network presenting greater cognitive deterioration.

The results are similar for TMT_A and TMT_B, where deterioration is indicated by high scores, and patients who had a poor family support network also showed greater deterioration.

Marital status.

We used a repeated measures ANOVA for independent samples to investigate whether a patient's marital status affected their cognitive performance.

Significant differences were obtained between women classified as Single (1), Married (2), Divorced (3), Widowed (4) in the following tests:

- Stroop word and colour before $(\mathrm{F}=3.384 ; \mathrm{p}<0.05)$,

- Stroop word and colour during $(\mathrm{F}=6.678 ; \mathrm{p}<0.001)$,

- Stroop word and colour after $(\mathrm{F}=4.565 ; \mathrm{p}<0.01)$.

Scheffé's Post Hoc or Multiple Comparisons test was used to find out between which groups there were differences in the various cognitive domains. The results showed that:

- For Stroop Word and Colour before, there were differences between women who were single and widowed $\left(1-4^{*}\right)$, with single women showing less cognitive deterioration.

- For Stroop Word and Colour during, the differences were between widows and the other three situations (4$1^{*}, 4-2^{*}$ and $\left.4-3^{*}\right)$, with widows presenting the greatest cognitive deterioration.

- For Stroop Word and Colour after, differences were found between widowed with single patients (4-1*), and widowed with married patients, with widowed women presenting the greatest cognitive deterioration.

Differences in the domains were also significant.

- TMT_A before ( $\mathrm{F}=23.367 ; \mathrm{p}<0.001)$,

- TMT_A during $(\mathrm{F}=21.550 ; \mathrm{p}<0.001)$,

- TMT_A after $(\mathrm{F}=21.656 ; \mathrm{p}<0.001)$,

- TMT_B before ( $F=19.617 ; \mathrm{p}<0.001)$,

- TMT_B during $(\mathrm{F}=18.745 ; \mathrm{p}<0.001)$,

- TMT_B after $(\mathrm{F}=17.585 ; \mathrm{p}<0.001)$.

Scheffé's Post Hoc or Multiple Comparisons test was used to find out between which groups there were differences in the various cognitive domains (in this case, greater cognitive deterioration is indicated by higher scores).

Significant differences were found between the widowed women and the patients in other situations, with greater cognitive deterioration being seen in the widowed group. 
On finding that the greatest cognitive deterioration was found in widowed women, we considered the possibility that there could be a relationship between marital status and the age of the patients. As was also found for previous objectives, when studying the differences by age groups, the greatest deterioration was found in older women. Because of this, we decided to study the relationship between the two variables using Contingency Tables and the Chi-squared test.

After statistically analysing the relationship between the two variables, our suspicion was confirmed, namely that there was a significant relationship (Chi-squared $=69.672 ; \mathrm{p}<0.001$ ) between marital status and age.

Finally, we examined whether years of schooling and employment status at the time of measurement influenced a patient's cognitive performance.

Years of Schooling.

To assess the influence that years of schooling had on the cognitive performance of the patients in our study, a repeated measures ANOVA for independent samples was run.

Statistically significant differences were obtained between women with basic (1), medium (2) and high (3) schooling levels in the following tests used to measure the different cognitive domains:

- Vocabulary before $(\mathrm{F}=3.524 ; \mathrm{p}<0.05)$;

- Vocabulary during $(\mathrm{F}=4.114 ; \mathrm{p}<0.05)$;

- Vocabulary after ( $\mathrm{F}=3.95 ; \mathrm{p}<0.05)$;

- Symbol search after ( $F=3.940 ; p<0.05)$;

- Letters and Number before ( $\mathrm{F}=6.897 ; \mathrm{p}<0.01)$;

- Letters and Number during $(\mathrm{F}=4.20 ; \mathrm{p}<0.05)$;

- Stroop word before $(\mathrm{F}=4.306 ; \mathrm{p}<0.05)$;

- Stroop word during $(\mathrm{F}=4.567 ; \mathrm{p}<0.05)$;

- Stroop word and colour before $(\mathrm{F}=8.677 ; \mathrm{p}<0.001)$;

- Stroop word and colour after $(\mathrm{F}=5.151 ; \mathrm{p}<0.01)$.

The differences were also significant for the following tests, where cognitive deterioration is indicated by higher scores:

- TMT_A before $(\mathrm{F}=23.728 ; \mathrm{p}<0.001)$,

- TMT_A during $(\mathrm{F}=23.253 ; \mathrm{p}<0.001)$,

- TMT_A after $(\mathrm{F}=22.445 ; \mathrm{p}<.001)$,

- TMT_B before $(\mathrm{F}=23.243 ; \mathrm{p}<0.001)$,

- $\quad$ TMT_B during $(\mathrm{F}=22.436 ; \mathrm{p}<0.001)$,

- TMT_B after $(\mathrm{F}=21.258 ; \mathrm{p}<0.001)$,

Scheffé's Multiple Comparisons or Post Hoc test was used to find out between which groups there were differences in the different cognitive domains. We found that, in the six cases above, there were significant differences between the three study level groups, with the greatest cognitive deterioration occurring in the basic education group, as shown in the following table.

Analysis of the data shows that patients with lower levels of schooling have worse cognitive performance in all the measures than those with higher levels of education. In addition, during and after the treatment, patients with a basic education level suffered greatest decline in cognitive function, compared to those with higher levels whose results were better.

Employment status.

The situation of the patients with regard to this variable changed during the treatment, so that:

- of the 79 women who were employed at the start of the treatment, only 33 were still working at the end of the treatment.

- of the 28 women who were unemployed at the start of treatment, the number was reduced to 29 after the end of treatment.

- 7 women were on temporary disability leave at the start of treatment; after the end of treatment, this figure was 53 patients.

- $\quad$ the number of retired patients stayed constant at 33 women.

- 4 patients were initially classified as "other"; after treatment, this number was reduced to 3.

The relationship between employment situation and cognitive performance was considered significant according to the results of the Chi-squared test $\left(\chi^{\wedge} 2=384.665 ; \mathrm{p}<0.001\right)$. 
When analysing the differences in the cognitive deterioration of the patients at the three measurement points, it was observed that the differences recorded prior to treatment were maintained once the treatment had been completed, in other words, patients who were actively working obtained higher initial scores which were maintained after treatment, compared to retired patients who obtained the lowest scores at the three measurement points.

We used a repeated measures ANOVA for independent samples to investigate whether a patient's employment status prior to starting treatment affected their cognitive performance.

Significant differences were detected between women classified as in active employment (1), unemployed (2), on temporary disability (3), and "other" (4) in the following tests measuring the cognitive domains studied:

\section{Discussion}

The results of our research show that there is no significant relationship between breast carcinoma type and the cognitive performance of the patients. Nor is there any evidence that the stage of the disease influences the cognitive functioning of the women.

After analysis of the data, it was observed that menopause negatively influences the cognitive performance of the oncology patients in our study, with postmenopausal women presenting the worst cognitive performance.

In a study of 117 women grouped into four categories: late reproductive stage (when women begin to notice changes in their menstrual period); early menopausal transition; late-stage menopausal transition; and early postmenopausal stage. Women in the early postmenopausal stage were found to perform less well in measures of verbal learning, verbal memory, and motor skills than women in the other three stages [15].

Some studies have postulated that radiotherapy may also increase the risk of cognitive impairment in cancer patients and, together with chemotherapy, increase cognitive deterioration [16,17].

Weis et al. suggest that, although cognitive changes may be subtle, these would impact memory, concentration, executive functions, and speed of information processing [18].

Shibamoto et al. report that cognitive changes are restricted to verbal memory only, and that attentional function and visual memory are not affected [19]. Along the same lines, Chang et al. found that, compared to nonradiated patients, patients who had received radiotherapy performed worse in the memory and learning domains [20].

The data from our investigation demonstrates that radiotherapy applied once chemotherapy has been completed does not influence the cognitive performance of the patients. However, this finding may be due to the fact that the final assessment in the study is just two months after the patients have completed their chemotherapy, in other words, any women who have been prescribed radiotherapy as an adjuvant treatment have had very few sessions of this. For that reason, the results on this aspect cannot be generalised.

Furthermore, most of the studies reviewed state that the cognitive impairments associated with radiotherapy usually appear in the long term, and although some studies report the possibility of these deficits appearing in early stages after radiotherapy, they are rarely severe at that time [21].

In the case of anaemia, our results show that lower haemoglobin levels in the blood negatively influence the cognitive performance of the patients. According to the study by Jacobsen, this variable is only related to cognitive performance when it varies below $12 \mathrm{~g} / \mathrm{dL}$. [22]. In other words, patients with anaemia will suffer more negative consequences than those with normal haemoglobin levels.

The influence of insomnia on the cognitive ability of the patients in our study was also studied, and it was found that quality of sleep does not influence cognitive performance.

There are no studies investigating the influence of insomnia on cognitive ability in cancer patients, but there are studies on the general population. These studies show that there is a relationship between insomnia and cognitive impairment. Most studies indicate how sleep disturbances, either in the form of nocturnal insomnia or excessive daytime sleepiness, may precipitate or aggravate cognitive problems [23].

We also examined whether certain sociodemographic variables might influence the cognitive performance under study. An analysis of the results showed that all of them were influential. However for some of them, such as marital status, it is not clear whether the deterioration was related to this variable or to age, since currently marital status does not define the true social situation of the patients in terms of family responsibilities, which may be an influential variable but perhaps difficult to measure, define, and objectively assess in order to draw scientific conclusions. Our research showed that widowed patients had worse cognitive performance than any of the other groups, but they were also the oldest. It is therefore unclear whether marital status has a relationship with increased cognitive deterioration, or whether it is age that is really exerting this influence. 
With respect to the years of schooling and employment status variables, we observed that patients with a higher academic level and those who were in employment had better cognitive performance in all the domains studied than the women who were not working and had less schooling. This seems to show that people who have good cognitive activity prior to treatment, in other words, who keep their brains active, perform better than those in the opposite situation.

This could mean that these variables exert a protective effect against chemotherapy-associated deterioration, although this does not imply that the cognitively active group cannot develop chemotherapy-associated impairment.

Age was shown to play a role in the cognitive performance of the patients in this study. We observed that the older the patient, the greater the degree to which chemotherapy affects cognitive performance, in other words, the greater the deterioration.

This influence may be associated with the neuronal depopulation that occurs with ageing, leading to poorer results in the tests used to assess cognitive performance [24].

As limitations of the study, we can comment on the individual differences that the individuals in the sample may present in terms of health. People can have other diseases that can condition the results. We think that being such a wide sample this limitation can be compensated.

\section{Conclusions}

From our results, we can conclude that factors such as sleep disorders, age, radiotherapy treatment, disease stage, and type of carcinoma do not affect the cognitive performance of oncology patients.

On the other hand, the presence of anaemia, menopause, the patient's support network and marital status, years of schooling and employment status do affect the cognitive performance of cancer patients.

Author Contributions: Conceptualization, All authors; methodology, M.I.R.G. and J.J.C.H.; software, E.J.F.R.; validation, B.R.M., C.S.G. and M.I.R.G.; formal analysis, E.J.F.R.; investigation, B.R.M.; resources, B.R.M.; data curation, C.S.G.; writingoriginal draft preparation, all authors; writing-review and editing, B.R.M. and E.J.F.R.; visualization, B.R.M.; supervision, M.I.R.G.; project administration, B.R.M.; funding acquisition, J.J.C.H. All authors have read and agreed to the published version of the manuscript.

Funding: This research received no external funding.

Institutional Review Board Statement: The study was conducted with the authorisation of the Clinical Research Ethics Committee of the Salamanca Health Area (ID:0000263), with the prior informed consent of the study subjects and in accordance with the Declaration of Helsinki. The participants were informed of the objectives of the project and the risks and benefits of the interventions that were carried out. Subject confidentiality was guaranteed at all times in accordance with the laws on the protection of personal data and biomedical research as established in the provisions of Organic Law 3/2018, of 5 December, on the Protection of Personal Data and guarantee of digital rights and Regulation (EU) 2016/679 of the European Parliament and of the Council of 27 April, 2016, on data protection (GDPR), and under the conditions established by Law 14/2007 on biomedical research. Name of the Council: Comité de Bioética de la Universidad de Salamanca (Bioethics Committee of the University of Salamanca). Board Affiliation: University of Salamanca.

Informed Consent Statement: Informed consent was obtained from all subjects involved in the study.

Data Availability Statement: The data can be consulted by any researcher by contacting the corresponding author directly.

Conflicts of Interest: The authors declare no conflict of interest.

\section{References}

1. Wefel J.S.; Saleeba A.K.; Buzdar A.U.; Meyers C.A.; Acute and late onset cognitive dysfunction associated with chemotherapy in women with breast cancer. Cancer. 2010, 116: 3348-56.

2. Wefel J.S.; Lenzi R.; Theriault R.L.; Buzdar A.U.; Cruickshank S.; Meyers C.A. “Che- mobrain” in breast cancer?. A prologue. Cancer. 2004, 101(3): 468-75.

3. Fernandez EJ, Sanchez C, Rihuete MI, Garcia B, Rodriguez A. Estudio sobre la influencia del tratamiento activo oncológico en la función cognitiva de los pacientes con cancer: chemobrain. Retocyl. 2018; 9: 19-34.

4. Rodriguez B, Fernandez EJ, Cruz JJ, Rihuete MI. Study of chemotherapy induced cognitive impairment in women with breast cancer. Int Jour Env Res Pub Health. 2020; 17(23): 88-92. 
5. Laser ablation after stereotactic radiosurgery: a multicenter prospective study in patients with metastatic brain tumors and radiation necrosis. J Neurosurg. 2018 May 4;130(3):804-811. doi: 10.3171/2017.11.JNS171273.

6. Survival outcomes in patients with recurrent glioblastoma treated with Laser Interstitial Thermal Therapy (LITT): A systematic review. Clin Neurol Neurosurg. 2020 Aug;195:105942. doi: 10.1016/j.clineuro.2020.105942.

7. Hermelink K.; Untch M.; Lux M.; Kreienberg R.;, Beck T.; Bauerfeind, I. et al. Cognitive function during neoadjuvant chemotherapy for breast cancer. Results of a prospective, multicenter, longitudinal study. Cancer. 2007,109(9): 1905-13.

8. Hurria A.; Goldfarb S.; Rosen C.; Holland J.; Zuckerman E.; Lachs M.S. et al. Effect of adjuvant breast cancer chemotherapy on cognitive function from the older patient's perspective. Breast Cancer Research and Treatment. 2006, 98: $343-48$.

9. Hurria A.; Rosen C.; Hudis C.; Zuckerman E.; Panageas K.S.; Lachs M.S. et al. Cognitive Function of Older Patients Receiving Adjuvant Chemotherapy for Breast Cancer: A Pilot Prospective Longutudinal Study. J Am Geriatr Soc. 2006, 54: 925-31.

10. Schagen S.B.; Hamburger H.L.; Muller M.J.; Boogerd W.; Van Dam F.M. Neurophysiological evaluation of late effects adjuvant high-dose chemotherapy on cognitive function. Journal of Neurooncology. 2001; 51: 159-65.

11. Vicente Pardo José Manuel, López-Guillén García Araceli. Problemas y factores psicológicos en el retorno al trabajo tras incapacidad temporal prolongada por cáncer de mama. Med segur trab. 2017; 63(248): 245-259.

12. Hermelink K.; Untch M.; Lux M.; Kreienberg R.; Beck T.; Bauerfeind, I. et al. Cognitive function during neoadjuvant chemotherapy for breast cancer. Results of a prospective, multicenter, longitudinal study. Cancer. 2007; 109(9): 1905-13.

13. Casavilca-Zambrano A, Custodio N, Cancino-Maldonado K, Liendo-Picoaga J, Vidaurre-Rojas T. Deterioro cognitivo por quimioterapia adyuvante en pacientes con cáncer de mama: Una problemática por definir en el Perú. Rev Neuropsiquiatr. 2021; 80(4): 239-246.

14. Padura I, Ulibarri A. Revisión crítica de la literatura sobre las necesidades no cubiertas de personas supervivientes de cáncer. Enf Onc. 2021; 23(1), 10-28.

15. Hilverda H.; Bosma I.; Heimans J.J.; Postma T.J.; Vandertop W.P.; Slotman B.J.; et al. Cognitive functioning in breast patients during radiotherapy and temozolomide treatment: Initial ndings. J Neurooncol 2010, 97: 89- 94.

16. Donovan K.A.; Small BJ, Andrykowski M.A.; Schmitt F.A.; Munster P.; Jacobsen P.B. Cognitive functioning after adjuvant chemotherapy and/or radiotherapy for early-stage breast carcinoma. Cancer. 2005; 104: 499-507.

17. Jalali R.; Singh S.; Budrukkar A. Techniques of tumour bed boost irradiation in breast conserving therapy: Current evidence and suggested guidelines. 2009. Acta Oncologica 46. 879-892.

18. Weiss B. Chemobrain: A translational challenge for neurotoxicology. Neurotoxicology. 2008.

19. Shibamoto Y.; Sugie C.; Iwata, H. Radio- therapy for metastatic brain tumors. Int J Clin Oncol 2009, 14, 281-8.

20. Chang E.L.; Wefel J.S.; Hess K.R.; Allen P.K.; Lang F.F.; Kornguth D.G. et al. Neurocogni- tion in patients with brain metastases trea- ted with radiosurgery or radiosurgery plus whole-brain irradiation: a randomised con- trolled trial. Lancet Oncol 2009; 10: 1037- 44 .

21. Welzel G.; Fleckenstein K.; Mai S.K.; Her- mann B.; Kraus-Tiefenbacher U.; Wenz F. Acute neurocognitive impairment during cranial radiation therapy in patients with intracranial tumors. Strahlenther Onkol. 2008 84 (12):647-54.

22. Jacobsen P.B.; Garland L.L.; Booth-Jones M.; Donovan K.A.; Thors C.L.; Winters E. et al. Relationship of haemoglobin levels to fatigue and cognitive functioning among cancer patients receiving chemotherapy. Journal of Pain and Symptom Management. 2004: 28, 7-18.

23. Medina-Chávez S.A.; Fuentes-Alexandro I.B.; Gil-Palafox L.; Adame-Galván F.; Solís-Lam L.Y.; Sánchez-Herrera. Guía práctica clínica. Diagnóstico y tratamiento del insomnio en el adulto mayor. Rev Med Inst Mex Seguro Soc., 52 (2014), pp. 108-119.

24. Sanchez C, Fernandez EJ. The effectiveness of a training programme in everyday cognition in healthy older adults: a randomised controlled trial. BMC Ger. 2021; 21(1): 79-92. 